\title{
Psychopathic traits associated with abnormal hemodynamic activity in salience and default mode networks during auditory oddball task
}

\author{
Nathaniel E. Anderson ${ }^{1} \cdot$ J. Michael Maurer ${ }^{1,2} \cdot$ Vaughn R. Steele $^{3} \cdot$ Kent A. Kiehl $^{1,2}$
}

Published online: 9 April 2018

(C) Psychonomic Society, Inc. 2018

\begin{abstract}
Psychopathy is a personality disorder accompanied by abnormalities in emotional processing and attention. Recent theoretical applications of network-based models of cognition have been used to explain the diverse range of abnormalities apparent in psychopathy. Still, the physiological basis for these abnormalities is not well understood. A significant body of work has examined psychopathy-related abnormalities in simple attention-based tasks, but these studies have largely been performed using electrocortical measures, such as event-related potentials (ERPs), and they often have been carried out among individuals with low levels of psychopathic traits. In this study, we examined neural activity during an auditory oddball task using functional magnetic resonance imaging (fMRI) during a simple auditory target detection (oddball) task among 168 incarcerated adult males, with psychopathic traits assessed via the Hare Psychopathy Checklist-Revised (PCL-R). Event-related contrasts demonstrated that the largest psychopathy-related effects were apparent between the frequent standard stimulus condition and a task-off, implicit baseline. Negative correlations with interpersonal-affective dimensions (Factor 1) of the PCL-R were apparent in regions comprising default mode and salience networks. These findings support models of psychopathy describing impaired integration across functional networks. They additionally corroborate reports which have implicated failures of efficient transition between default mode and task-positive networks. Finally, they demonstrate a neurophysiological basis for abnormal mobilization of attention and reduced engagement with stimuli that have little motivational significance among those with high psychopathic traits.
\end{abstract}

Keywords Psychopathy $\cdot$ Attention $\cdot$ Oddball $\cdot$ fMRI $\cdot$ Salience Network $\cdot$ Default Mode Network

Psychopathy is a multifaceted disorder characterized by a combination of personality traits, behavioral patterns, and cognitive features (Cleckley, 1941; Hare, 2003). These include chronic antisocial behavior, lack of empathy, and an unscrupulous, manipulative interpersonal style. Psychopathy

Electronic supplementary material The online version of this article (https://doi.org/10.3758/s13415-018-0588-2) contains supplementary material, which is available to authorized users.

Nathaniel E. Anderson

nanderson@mrn.org

1 The Nonprofit Mind Research Network (MRN) \& Lovelace Biomedical and Environmental Research Institute (LBERI), 1101 Yale Blvd NE, Albuquerque, NM 87106, USA

2 University of New Mexico, Albuquerque, NM, USA

3 Neuroimaging Research Branch, National Institute of Drug Abuse, Intramural Research Program, National Institutes of Health, Baltimore, MD, USA is further characterized by deficits in affective processing (Blair, 2005; Kiehl, 2006) and abnormalities in attention that hinder flexible distribution of cognitive resources (Newman, 1998). These distinctive features of psychopathy set this disorder apart from antisocial behavior per se (Blair, Peschardt, Budhani, Mitchell, \& Pine, 2006; Frick \& White, 2008) and remain a prominent focus of related translational research.

Neural dysfunction associated with psychopathy has been observed in paralimbic brain regions responsible for salience detection, emotional processing, and the integration of this information into higher-order processing (Anderson \& Kiehl, 2012). As such, well-established models of psychopathy have tended to focus on deficits in emotional processing; however, these deficits have not fully accounted for the neurocognitive abnormalities recognized in this disorder. Recent evidence suggests that abnormalities in selective attention among psychopaths interfere with their adaptive redistribution of attention, hindering adaptation to new and potentially relevant environmental cues once a primary focus of attention has been established (Baskin-Sommers, Curtin, \& 
Newman, 2011; Baskin-Sommers, Curtin, Li, \& Newman, 2012). Newman's Response Modulation Theory suggests that this deficit is sufficient to impede processing of contextual emotional information under circumstances where emotional information competes with some other primary focus of attention (Newman, Curtin, Bertsch, \& Baskin-Sommers, 2010; Baskin-Sommers, Curtin, \& Newman, 2013). Evidence for this attention-based model of psychopathy is compelling; however, due to limited neuroimaging work addressing attention in psychopathy, there remains much to be learned about the specific neurocognitive properties that support these limitations. A recent theoretical approach, the Impaired Integration model, appeals to general information processing abnormalities dependent on large-scale intrinsic network function (Hamilton, Hiatt Racer, \& Newman, 2015). This model suggests specific impairments in interactions between discrete networks that dominate different forms of information processing, including the default mode network and salience network, which overlap with regions implicated in paralimbic dysfunction, including medial prefrontal cortex, amygdala, anterior cingulate, posterior cingulate, and insula. Still, relatively little work has demonstrated physiological evidence for these specific deficits, especially during basic cognitive tasks in the absence of high affective content.

Neurocognitive features of attention can be examined using oddball, target-detection tasks (Herrmann \& Knight, 2001). Oddball tasks involve discrimination of rare target stimuli among a background of more frequent nontarget (standard) stimuli. These tasks sometimes include infrequent novel, nontarget distractors intended to elicit evaluation of changing stimulus features but which also require response-inhibition. Oddball tasks are commonly employed when examining attention-related neurocognitive features of psychiatric disorders, and most often these studies have relied on event-related potentials (ERPs) derived from electroencephalographic techniques.

A number of electrocortical abnormalities have commonly been associated with psychopathy during simple target detection tasks, largely reserved to neural responses for target and novel stimuli (see Gao \& Raine, 2009 for review). Our research team recently exposed a previously unreported psychopathy-related abnormality during engagement with frequent standard stimuli in an oddball task. Specifically, psychopathic traits were associated with reduced amplitude in the late, positive slow-wave component elicited by frequent, nontarget (standard) stimuli (Anderson, Steele, Maurer, Bernat, \& Kiehl, 2015). This late slow-wave component is generally associated with sustained evaluative processing following response selection (García-Larrea \& Cézanne-Bert, 1998), which may be limited among psychopaths. Because this effect accompanied a nontarget stimulus, we suggested that this may be a novel marker for basic attention-related abnormalities, as psychopathy is characterized by diminished engagement with stimuli that are not of immediate motivational significance (Newman, 1998).

ERP measures are endowed with high precision in the time domain; however, they are severely limited in their capacity to address spatial patterns revealing functional anatomy and network-related brain activity (see Grech et al., 2008 for further discussion). Supplementing ERP studies with other domains of physiological recordings, including event-related fMRI, is a helpful way to understand more about the nature of some ERP signals. For example, the widely studied P3 ERP component (associated with target detection) results from the summed activity of neurons in widely distributed brain regions, which are not individually necessary for successful target detection or generation of the potential itself (Polich \& Squire, 1993). Intracranial recordings and functional neuroimaging have demonstrated at least 38 differentiable brain areas that contribute to the generation of the $\mathrm{P} 3$ and target detection (Halgren, Marinkovic, \& Chauvel, 1998; Kiehl et al., 2005). Neurophysiological evidence suggests that P3 coincides with widespread, phasic noradrenergic neuromodulation (Nieuwenhuis et al., 2005). Its amplitude and latency change within-subjects under varying circumstances, such as task complexity, memory load, and even biological states (Polich, 1987; Polich \& Kok, 1995). As such, the P3 (and other ERP components) are not unitary traits but rather a measure representing several overlapping cognitive mechanisms (Pfefferbaum, Ford, \& Kraemer, 1990). Event-related fMRI measures can thus provide helpful information about brain regions and networks involved in ERP effects (see also Calhoun, Adali, Pearlson, \& Kiehl, 2006).

To date, only one study has investigated an oddball paradigm in relation to psychopathy using fMRI. Juárez and colleagues used Independent Component Analysis (ICA) of fMRI data to define networks of functionally related neural activity among inmates evaluated for psychopathy (Juárez, Kiehl, \& Calhoun, 2013). ICA is a technique that identifies unique sources of variance in complex, noisy systems, incluiding fMRI data (Calhoun, Kiehl, \& Pearlson, 2008). Juárez et al. found that psychopathic traits were positively correlated with an ICA-derived component spatially consistent with the default mode network. The default mode network (DMN) refers to a network of brain regions highly active during resting-state, task-free, introspective processes including medial prefrontal cortex, posterior cingulate/precuneus, and temporoparietal junction (Greicius, Krasnow, Reiss, \& Menon, 2003; Raichle et al., 2001). The power of DMN activity decreases during target detection in balance with discrete task-positive networks that are brought online (Cocchi, Zalesky, Fornito, \& Mattingley, 2013). In another investigation, individuals diagnosed with psychopathy demonstrated failures to deactivate appropriately DMN during a simple Go/No-Go task (Freeman et al., 2015). These findings may suggest failures in the systematic coupling and decoupling of 
networks that promote effective signal processing and basic elements of cognition (see also Philippi et al., 2015; Pujol et al., 2011, Sheng, Gheytanchi, \& Aziz-Zadeh, 2010). Further, they may represent functional anatomical correlates of ERP abnormalities during sustained slow-wave activity previously reported. In neurotypical individuals, dynamic shifts between DMN-dominant states and several other discrete cognitive networks (e.g., executive control, salience network) provides a physiological basis for natural shifts of attention (Fox et al., 2005). This is a compelling context from which to evaluate attention-based models of psychopathy.

Some level of interpretation is left ambiguous by ICA analysis, because this data-driven approach does not assume an $a$ priori structure to the data and thus does not allow for the direct comparison of traditional univariate contrast elements defined by task conditions (e.g., neural activity that is greater during the target condition than during the standard condition). Examining these univariate contrasts can reveal condition-bound specificity and directionality of effects that are not obvious in alternative multivariate, data-driven approaches. Furthermore, contrast-based GLM analyses have important implications for the interpretation of extant and future traditional fMRI studies of psychopathy, especially if abnormalities are revealed in conditions often used as a baseline for examining task-based effects in functional imaging.

The purpose of this study was to examine psychopathyrelated abnormalities in hemodynamic response during an auditory oddball task. Using a contrast-based general linear model (GLM) approach for fMRI analyses, we were able to examine activity related to many specific event-related effects previously reported in ERP literature. Based on our recent work examining ERP effects with an identical task among incarcerated individuals (Anderson et al., 2015), we anticipated psychopathy-related hemodynamic differences during the frequent standard stimulus. Because individual ERP components do not easily translate to the prolonged temporal dynamics of BOLD response, detailed directional functionalanatomical relationships could not justifiably be expected and were not specified, a priori. Based on accumulating literature revealing unique correlates of emotional/interpersonal elements and lifestyle/behavioral elements of psychopathy, we expected factor and facet scores on the PCL-R to reveal more specific directional and more stable effects than those associations with PCL-R total scores. Factors and facets of the PCL-R are associated with dimensional traits that have often revealed discrete (sometimes opposing) relationships with physiological measures (see for example Anderson et al., 2015; Juárez et al., 2013; Maurer et al., 2015; Philippi et al., 2015; Steele et al., 2016; Wolf et al., 2015), however, there has been limited prior work extending these findings to basic cognitive tasks using neuroimaging techniques. To our knowledge, this is the first report to examine relationships between psychopathic traits and specific functional-anatomical dynamics during an oddball target detection task using event-related GLM analysis of fMRI.

\section{Method}

\section{Participants}

Volunteers were recruited via advertisements and word-ofmouth at two medium-security state correctional facilities in New Mexico. Participants were compensated with an hourly wage commensurate with the rate of pay for labor at their respective correctional facilities. Volunteers provided their written, informed consent, and all procedures were approved by the University of New Mexico Health Science Center Institutional Review Board. Adult male inmates $(\mathrm{n}=222)$ completed an auditory oddball fMRI protocol as well as clinical interviews. Due to the co-occurrence of many potentially confounding variables common among incarcerated individuals, great care must be taken in the selection/exclusion of participants. Participants were excluded if they met DSMIV-TR diagnostic criteria for schizophrenia, bipolar, current major depression, anxiety disorder (e.g., panic, PTSD), or attention deficit disorder $(n=12)$. An additional eight participants were excluded for a history of serious and/or repeated head injury resulting in prolonged loss of consciousness. We also excluded three participants with estimated IQs below 70 . Behavioral performance was carefully monitored during the task; due to the ease of the task, poor performance often can be attributed to fatigue and subsequent lapses of attention during the scan session. Participants $(n=26)$ were removed for poor behavioral performance on the task including low number of hits $(<15)$ or high number of false alarms $(>10)$. Finally, five participants were excluded due to excessive movement and/or uncorrectable loss of whole brain coverage during scanning procedures.

The final sample for the present analyses included 168 adult male inmates. Ages ranged from 19 to 66 years $(\mathrm{M}=$ 37.1, $\mathrm{SD}=11.2)$. Ethnicity and race were self-reported: 87 (51.7\%) white/Caucasian, 19 (11.3\%) American Indian/ Alaska native, 18 (10.7\%) black/African American, 40 (23.8\%) other/multiple, and 4 (2.3\%) chose not to provide this information; 59 self-identified as Hispanic/Latino and 109 as non-Hispanic.

\section{Assessments}

Psychopathy was assessed using the Psychopathy ChecklistRevised (PCL-R; Hare, 2003). This is an expert-administered rating scale consisting of a semi-structured interview and an extensive file review to provide collateral information for scoring participants on 20 items. Each item is rated on a three-point scale: 0 indicated no evidence, 1 indicated some 
evidence, and 2 indicated pervasive evidence in many domains of an individual's life. Of a maximum of 40 points, a score of 30 or higher is the recommended cutoff for a diagnosis of psychopathy. These scores also provide dimensional assessments of psychopathic traits (Hare \& Neumann, 2005). These traits are divided into two major factors. Factor 1 indicates interpersonal (facet 1) and affective (facet 2) traits, such as grandiosity and a lack of empathy and remorse. Factor 2 indicates lifestyle (facet 3) and antisocial (facet 4) traits, including impulsiveness and long-range developmental patterns of behavioral problems. PCL-R total scores in the current sample ranged from 7 to $37.9(\mathrm{M}=21.3, \mathrm{SD}=6.6)$.

Psychometric properties of the PCL-R have been comprehensively vetted in prior literature (Hare, 2003; Hare \& Neumann, 2006). For this study, all interviews were conducted by staff who have undergone extensive training and supervision. All interviews were videotaped, and a portion of these are double-rated to ensure consistency and avoid rater drift. Interrater reliability for our trained staff is 0.96 (Intraclass Coefficient).

Diagnoses for other psychiatric disorders were based on criteria from the Structured Clinical Interview for DSM-IVTR Axis I disorders - Patient Edition (SCID I-P; First, Spitzer, Gibbon, \& Williams, 2002). All administers are extensively trained and vetted during supervised and videotaped sessions. Trained staff demonstrate high diagnostic accuracy and interrater reliability (Ventura, Liberman, Green, Shaner, \& Mintz, 1998). SCID diagnoses were primarily used for exclusionary purposes as noted above. Participants were not excluded for substance dependence, because substance misuse is highly comorbid with psychopathy and occurs at high rates among incarcerated individuals in general.

As discussed in more detail below, two statistical modeling procedures are applied to compare the effects of psychopathy scores alone and separate models that include several covariates. Covariates include the number of substance dependencies each participant met SCID I-P criteria for to model variation due to lifetime substance use. This method of accounting for substance use in incarcerated samples has been used in several previous publications (Cope et al., 2014; Ermer, Cope, Nyalakanti, Calhoun, \& Kiehl, 2012). The number of identified substance dependence diagnoses ranged from 0 to 6 $(\mathrm{M}=1.4, \mathrm{SD}=1.5)$. IQ estimates were calculated using the vocabulary and matrix reasoning subtests of the Wechsler Adult Intelligence Scale III (WAIS-III; Wechsler, 1997). IQ estimates ranged from 72 to $148(\mathrm{M}=98.8, \mathrm{SD}=13.9)$.

\section{Oddball task description}

Participants performed an auditory oddball task while undergoing fMRI data collection. The task was programmed and implemented via Presentation software v.16.3 (NeuroBehavioral Systems, www.neurobs.com). Task parameters are identical to prior work utilizing this task in healthy controls (Kiehl et al., 2005). Stimuli were presented over two experimental runs with 244 stimuli, delivered through MR-compatible, $30 \mathrm{~dB}$ sound-attenuating headphones. Auditory stimuli consisted of frequent standard lowpitched $(1,000 \mathrm{~Hz})$ tones $(80 \%)$, infrequent target highpitched $(1,500 \mathrm{~Hz})$ tones $(10 \%)$, and infrequent nontarget, novel stimuli (10\%), which were computer-generated sounds (static, bells, beeps, varied in tone). Each stimulus was presented for $200 \mathrm{~ms}$ in pseudorandom order with an interstimulus interval ranging from $500-2,100 \mathrm{~ms}$. Target and novel stimuli were always preceded by at least three standard stimuli (range 3-5). Intervals between target and novel stimuli ranged from 8-12 seconds. Participants were instructed to respond quickly and accurately to high-pitched, target tones by pressing a single button on an MRI-compatible response box with their right index finger. They were instructed not to respond to any of the other stimuli. Before beginning the task, each participant performed a practice session of ten trials to ensure their understanding of the task. Reaction times were computed on trials for which the participant responded correctly within 1,500 ms post-stimulus. Omission errors included any missed target tones or any response with a latency of greater than $1,500 \mathrm{~ms}$ following the onset of the target stimulus. Errors of commission were defined as responses following the frequent standard or novel stimuli within $1,500 \mathrm{~ms}$ of stimulus onset.

\section{MRI data collection and processing}

fMRI data were acquired onsite at the prison facilities, using a mobile Siemens Avanto 1.5 Tesla MR scanner equipped with advanced SQ gradient engine (max slew rate, $200 \mathrm{~T} / \mathrm{m} / \mathrm{s} ; 346$ $\mathrm{T} / \mathrm{m} / \mathrm{s}$ vector summation, rise time $200 \mu \mathrm{s}$ ) and a 12 -element head coil. Conventional spin-echo T1 weighted sagittal localizers were acquired for use in prescribing the functional image volumes. The echoplanar image gradient-echo pulse sequence (TR $2000 \mathrm{~ms}$, TE $39 \mathrm{~ms}$, FA $75^{\circ}$, FOV $24 \times 24$ $\mathrm{cm}, 64 \times 64$ matrix, $3.8 \times 3.8 \mathrm{~mm}$ in plane resolution, 4-mm slice thickness, 27 slices) effectively covers the entire brain in 2 seconds. Head motion was limited using padding and restraint. Functional images were reconstructed offline at 16-bit resolution and manually reoriented to the anterior commissure/posterior commissure line. MRI data were processed using SPM software. Motion correction was achieved using the ArtRepair Toolbox (Mazaika et al., 2007), effectively detecting and removing severe motion-related artifacts. Images were motion-corrected using INRIalign, an algorithm unbiased by local signal changes (Freire \& Mangin, 2001; Freire et al., 2002). Six realignment parameters ( 3 translations and 3 rotations) were entered as covariates in the statistical models in order to remove additional variance due to movement. Functional images were spatially normalized to the 
Montreal Neurological Institute (MNI) template using a transformation with both linear and nonlinear components (Friston et al., 1995). Normalized data were smoothed (12 $\mathrm{mm}$ FWHM) and a high pass filter (cutoff: 1/128 s) removed low frequency noise (e.g., respiratory artifact, scanner drift).

Event-related responses to target, novel, and standard stimuli were modeled using a synthetic hemodynamic response function composed of two gamma functions. The first gamma function modeled hemodynamic response using a peak latency of $6 \mathrm{~s}$. A term proportional to the derivative of this gamma function was included to allow for small variations in peak latency. The second gamma function and associated derivative was used to model the small overshoot of the hemodynamic response on recovery (Calhoun, Stevens, Pearlson, \& Kiehl, 2004).

For each participant, images representing the hemodynamic response for contrasts of interest were computed. Contrasts include (1) target stimuli relative to the frequent standard stimuli, (2) novel stimuli relative to the frequent standard stimuli, and (3) standard stimuli relative to an implicit/residual baseline (calculated as the variance in the time series not otherwise explicitly modeled). One-sample $t$-tests in SPM were used to detect main effects for each contrast. Multiple regression analyses were used to evaluate the unique contribution of psychopathy scores to variation in hemodynamic response for each contrast. Separate models were used to examine the effect of PCL-R total score, PCL-R Factor 1, and Factor 2 scores and the four PCL-R facet scores in their own models. Additional models examined the effects of PCL-R scores when including covariates accounting for effects for age, substance dependence, and the other respective factors and facets to evaluate more precisely the unique variance attributable to each individual factor/facet.

Choosing a baseline Modeling event-related hemodynamic response in fMRI using the general linear model is intrinsically contrast-driven. That is, BOLD signal ultimately represents net differences in hemodynamics between two conditions, and these conditions need to be carefully considered when interpreting results. The comparison condition for any given contrast is often regarded only as a baseline and rarely plays a major role in interpreting the effect vis-à-vis the primary condition of interest (e.g., targets). However, different baselines have the potential to eliminate or even reverse the direction of some BOLD effects (Stark \& Squire, 2001).

For target detection tasks, the use of an implicit baseline (time otherwise un-modeled in the task) or alternatively the frequent standard condition as a baseline typically yield very similar results (Kim, 2013). Our work with healthy samples using an identical task to the present one has also demonstrated similar findings (Kiehl et al., 2005; Stevens, Laurens, Liddle, \& Kiehl, 2006). In this work, we report analyses using the frequent standard condition for isolating cognitive activity related to target detection and evaluation of unexpected, novel events, factoring out the influence of primary auditory processing; however, we also examined this activity using the implicit baseline (cf. Kiehl et al., 2005). Because this work is predicated by suggestions that baseline activity during frequent standard stimuli may be affected in psychopathy (Anderson et al., 2015), the direct comparison of the frequent standard condition and the implicit baseline is very important. We model the frequent standard condition against the implicit baseline to directly demonstrate these differences.

Regions of interest Regions of interest (ROIs) were identified from previously published work examining whole-brain effects from an identical auditory oddball task in 100 healthy controls (Kiehl et al., 2005; see also Kiehl \& Liddle, 2001). These studies identify 38 ROIs for response to Targets (Targets greater than Standards), 33 ROIs for response to Novels (Novels greater than Standards), and 3 ROIs for response to Standards (greater than the residual baseline). Coordinates for these ROIs are identified and labelled in our results tables (Tables 2, 3 and 4). Main effects (disregarding psychopathy) were examined to verify significant hemodynamic change in these ROIs for the present sample. ROIs for analysis were created using Wake Forest University PickAtlas (Maldjian, Laurienti, Kraft, \& Burdette, 2003) and categorized with the automated anatomical labeling (AAL) atlas (TzourioMazoyer et al., 2002). Each ROI was then examined for associations between hemodynamic response and PCL-R scores. For the contrasts of interest, activity in each ROI was defined as the peak signal in an 8-mm sphere surrounding the center coordinates in MNI space, controlling for multiple comparisons by applying a correction for False Discovery Rate (FDR) (Genovese, Lazar, \& Nichols, 2002). PCL-R total, factor, and facet scores were examined in separate models, each alone in the model. Then, for comparison, additional models examined the effects of PCL-R scores when including covariates accounting for effects for age, substance dependence, and the other respective factors and facets to evaluate more precisely the unique variance attributable to each individual factor/facet.

Whole brain effects Abnormal hemodynamic effects related to psychopathy may not be limited to the most prominent brain areas identified among in neurotypical individuals for each contrast. We followed ROI analyses with an examination of hemodynamic effects correlated with PCL-R scores across the entire brain. Significant effects in these analyses represent unique event-specific hemodynamic effects related to psychopathy in contrast to incremental variation in ROIs identified via neural activity among healthy controls. For these analyses, we apply more stringent FDR adjustments for anticipated false discoveries across the expanded search area of the entire brain. 
Table 1. Zero-order correlations between PCL-R scores, assessment variables, and behavioral performance

\begin{tabular}{llllllll}
\hline & Age & IQ & Subst. Dep. & Hit RT & \# Hits & \#Novel FA & \#Standard FA \\
\hline PCL-R Total & -0.127 & -0.005 & $0.245^{* *}$ & $-0.165^{*}$ & 0.010 & -0.047 & -0.005 \\
PCL-R Factor 1 & 0.018 & -0.002 & 0.143 & $-0.172^{*}$ & 0.053 & -0.057 & 0.011 \\
PCL-R Factor 2 & $-0.294^{* *}$ & -0.012 & $0.338^{* *}$ & -0.093 & -0.071 & -0.029 & 0.018 \\
\hline
\end{tabular}

${ }^{*} p \leq 0.05 ; * p \leq 0.001$. Subst. Dep. $=$ Substance Dependence as defined in methods. Hit RT $=$ Reaction time for hits. \#Hits $=$ Number of target hits. \#Novel FA $=$ number of false alarms on novel stimuli. \#Standard FA = number of false alarms on frequent standard stimuli

\section{Results}

\section{Behavioral performance}

As noted, the target detection task is designed to maintain high behavioral performance in a wide variety of clinical samples. On average, participants missed only one target across both runs ( $>98 \%$ correct responses). Participants averaged less than two false alarms to novel distractors and less than 1 false alarm for frequent standard stimuli. Response accuracy was not significantly associated with PCL-R scores or other subject variables (age, substance dependence, IQ). Reaction times for target stimuli $(\mathrm{M}=500.4 ; \mathrm{SD}=78.5)$ showed modest but significant correlations with PCL-R total and Factor 1 scores, such that higher scorers had faster response times to targets (Table 1).

\section{Main effects: targets, novels, standards}

All ROIs previously identified for targets (38 regions) and novel stimuli (33 regions) showed significant activation across all subjects with $t$-values ranging from 5.89 to 27.25 (Tables 2 and 3; Figure 1). For the standard > baseline condition, ROIs corresponding to left and right superior temporal gyrus (surrounding primary auditory cortex) were highly active ( $t$-values of 15.03 and 18.55 respectively; Table 4; Figure 1). There was no significant main effect for the anterior cingulate ROI as identified in Kiehl et al. (2005) in the standard > baseline condition in healthy control participants. Notably, however, the anterior cingulate region exhibits a significant interaction with PCL-R (discussed further below), which may account for lack of replication in main effects.

\section{Psychopathy-related effects}

Target stimuli Examining ROIs for targets $>$ standards, there were no significant correlations with PCL-R total score when it was entered by itself into the regression equation or when accounting for covariates of age and substance dependence. Individual factors and facets of the PCL-R demonstrated sparse significant correlations, mostly positive associations (Table 5); however, none of these effects survived thresholds applying corrections for multiple tests (38 ROIs, $p<0.0013$ ).
When using an implicit baseline (instead of the standard stimulus), the sparse psychopathy-related effects for targets $>$ baseline were only somewhat diminished, but still no psychopathy-related effects survived corrections for multiple comparisons. When performing whole-brain analyses, no significant associations between PCL-R scores and BOLD signal for this contrast survived FDR correction.

Novel stimuli Examining ROIs for novels $>$ standards, several trends are apparent for PCL-R total, factor, and facet scores (Table 6); however, few survive conservative corrections for multiple comparisons (33 ROIs; $p<0.0015$ ). Effects surviving this threshold exclusively include a positive association between PCL-R facet 4 scores and BOLD signal in the medial frontal gyrus and dorsal anterior cingulate, only when controlling for substance dependence, age, and the combined effects of other facet scores. When using an implicit baseline (instead of the standard stimulus), effects for novels $>$ baseline remained largely the same. No psychopathy-related effects survived corrections for multiple comparisons. When searching the whole brain, no additional associations were discovered that survive FDR correction.

Standard vs. Baseline Examining ROIs for standards $>$ baseline, significant associations with PCL-R total, factor, and facet scores were apparent (Table 7), many surviving corrections for multiple comparisons (3 ROIs; $p<0.017$ ). Alone in the model, PCL-R facet 3 scores were negatively correlated with BOLD signal in the ACC. Factor 1 scores were negatively correlated with activation in bilateral superior temporal gyrus. When controlling for covariates of age, substance dependence, and the effects of other factors, opposing associations are evident for Factor 1/Facet 2 (negative) and Factor 2/Facet 4 (positive) in the superior temporal gyrus. A similar trend of opposing directional effects is evident in the ACC. Importantly, only negative associations between Factor and Facet scores of the PCL-R survive corrections for multiple tests.

Examining whole brain associations reveal widespread negative correlations between PCL-R scores and hemodynamic response for Standards vs. Baseline that survive global FDR-correction across the entire brain. These associations were strongest with PCL-R Factor 1 scores (Figure 2). 
Table 2. Target Stimuli Main Effects in ROIs

MNI coordinates

$t$-value (peak)

$\begin{array}{ll}\begin{array}{ll}\text { Frontal lobes } \\ 1 .\end{array} & \\ 2 . & \text { L. superior middle frontal gyrus } \\ 3 . & \text { R. superior middle frontal gyrus } \\ 4 . & \text { L. middle frontal gyrus } \\ 5 . & \text { L. inferior frontal gyrus } \\ 6 . & \text { L. inferior frontal gyrus } \\ 7 . & \text { R. inferior frontal gyrus } \\ 8 . & \text { Medial frontal gyrus } \\ 9 . & \text { Anterior cingulate gyrus } \\ 10 . & \text { L. insula } \\ 11 . & \text { R. insula } \\ 12 . & \text { L. precentral gyrus } \\ 13 . & \text { L. precentral gyrus } \\ \text { Part } & \text { R. precentral gyrus }\end{array}$

Parietal lobes

14.

15.

16.

17.

18.

19.

20.

21.

22.

Temporal lobes

23.

24.

25.

26.

27.

28.

29.

Occipital lobe

30.

31.

Deep gray

32.

33.

34.

35.

36.

Cerebellum

37.

38.
L. postcentral gyrus

L. postcentral gyrus

R. postcentral gyrus

L. superior parietal lobule

R. superior parietal lobule

L. inferior parietal/supramarginal gyrus

R. inferior parietal/supramarginal gyrus

Posterior cingulate gyrus

Precuneus

L. superior temporal gyrus

L. middle temporal gyrus

R. middle temporal gyrus

L. inferior temporal gyrus

R. inferior temporal gyrus

L. amygdala/parahippocampal gyrus

R. amygdala/parahippocampal gyrus

L. lingual gyrus/cuneus

R. lingual gyrus/cuneus

L. thalamus/caudate

R. thalamus/caudate

L. putamen/globus pallidus

R. putamen/globus pallidus

Brainstem

$\mathrm{x}$
-36
24
-40
-56
-56
52
0
0
-48
44
-36
-36
28

$-56$

$-32$

60

$-36$

24

$-60$

56

0

0

$-48$

$-60$

60

$-56$

60

$-24$

24

$-8$

16

$-16$

8

$-32$

32

0

\section{y}

44

52

40

0

24

8

8

(2)

12

12

16

$-16$

$-32$

$-4$

\section{$-24$}

$-36$

$-28$

$-40$

$-52$

$-36$

$-36$

$-48$

$-36$

$-32$

$-60$

$-28$

$-8$

$-56$

0

4

$-68$

$-68$

$-20$

$-16$

$-4$

8

$-24$

$-28$

20

$-60$

$-52$
Z

24

24

4.83

17.14

14.67

17.79

6.50

21.12

25.14

25.21

20.23

20.91

24.18

24.04

14.33

60

26.72

22.56

22.73

22.47

10.03

24.82

22.80

6.91

12.09

17.48

14.85

17.39

21.32

15.99

17.68

18.50

$-12$

$-12$

20.50

13.66

4

19.48

18.80

20.46

23.06

18.68

$-24$

21.70

$-32$

21.70

27.25

Threshold for $p<0.05$ corrected for FDR is $t=1.69 ; p<0.01 t=2.38 ; p<0.001 t=3.18$

Controlling for age, substance dependence, and Factor 2 scores, 30 significant clusters were identified across the whole brain including frontal, parietal, temporal, limbic, occipital, subcortical, and cerebellar regions. Peak $t$-values within 
Table 3. Novel stimuli main effects in ROIs

MNI coordinates $t$-value (peak)

Frontal lobes

1. L. middle/inferior frontal gyrus $\quad \begin{array}{lll}-52 & 12 & 32\end{array}$

$\begin{array}{llllll}2 . & \text { R. middle/inferior frontal gyrus } & 48 & 16 & 28 & 16.44\end{array}$

3. L. inferior frontal gyrus/insula $\quad-44 \quad 20 \quad-12 \quad 11.72$

$\begin{array}{llllll}\text { 4. R. inferior frontal gyrus/insula } & 48 & 24 & -8 & 16.57\end{array}$

5. Medial frontal gyrus $\quad \begin{array}{lllll}0 & 24 & 36 & 12.80\end{array}$

$\begin{array}{llllll}\text { 6. } & \text { L. anterior cingulate gyrus } & 0 & 16 & 32 & 9.58\end{array}$

$\begin{array}{llllll}7 . & \text { R. anterior cingulate gyrus } & 4 & 28 & 32 & 11.90\end{array}$

8. L. insula

9. R. precentral gyrus

Parietal lobes

10. L. postcentral gyrus

11. R. postcentral gyrus

12. L. postcentral gyrus

13. L. superior parietal lobule

14. R. posterior cingulate gyrus

15. L. inferior parietal lobule

16. R. inferior parietal lobule

Temporal lobes

17. L. superior temporal gyrus

18. R. superior temporal gyrus

19. R. middle temporal gyrus

20. R. middle temporal gyrus

21. L. inferior temporal gyrus

22. R. inferior temporal gyrus

23. L. amygdala

24. R. amygdala

Occipital lobe

25. L. lingual gyrus/cuneus

26. R. lingual gyrus/cuneus

Deep Gray

27. L. thalamus/caudate

28. R. thalamus/caudate

29. Brainstem

Cerebellum

30. L. cerebellum

31. L. cerebellum

32. R. cerebellum

33. R. cerebellum

Threshold for $p<0.05$ FDR corrected $t=1.91 ; p<0.01 t=2.59$; $p<$ $0.001 t=3.38$

clusters ranged from 2.3 to 5.17 , and 25 of the 30 regions survive FDR-corrected whole-brain thresholds at $p<0.005$ (Table 8). Examining facets, these effects are apparently strongest for Facet 2, especially in anterior temporal, insula, and lateral prefrontal areas; however, facet-level effects do not survive in models that covary other facets, presumably due to strong correlations between facets. Correlations with
Factor 1 are represented as these effects are the most stable with respect to covariates.

Supplementary analysis Some prior studies have indicated potential ethnic/racial differences in the manifestation of psychopathic traits as well as occasional failures replicating neurophysiological effects among non-European Americans (see Sullivan \& Kosson, 2006 for review). Approximately half of the current sample did not identify as Caucasian. We examined these group differences in psychopathy scores as well as hemodynamic effects. There were minor differences in PCL-R Factor 2 scores and Facet 4 scores. Caucasians scored slightly lower on Factor 2 (mean $=11.72, \mathrm{SD}=3.76)$ than minorities $($ mean $=12.96, \mathrm{SD}=3.87), t=2.10 ; p=0.038$, and slightly lower on Facet 4 scores $($ mean $=6.33, \mathrm{SD}=2.39)]$ than minorities $($ mean $=7.11, \mathrm{SD}=2.45), t=2.03 ; p=0.044$. In the three primary contrasts germane to this study, there were no significant differences between groups in activations across the brain after applying corrections for false discovery rate (FDR). Examining average beta values (in 8-mm spheres) for contrasts demonstrating associations with psychopathic traits (Tables 5, 6, 7 and 8), $t$-tests revealed no group differences which survived corrections for multiple comparisons. The evidence from these analyses suggests overall stability of these effects across Caucasian and minority groups in our sample.

\section{Discussion}

This study was designed to examine differences in fMRI BOLD response attributable to psychopathic traits during an auditory oddball task. As expected, individual factors and facets of the PCL-R revealed distinct relationships with BOLD signal in regions associated with target detection and novel stimuli. Furthermore, the largest, most stable psychopathy-related effects were apparent when examining the frequent standard condition against an implicit baseline (Anderson et al., 2015). Specifically, Factor 1 scores on the PCL-R were associated with widespread, reduced BOLD signal in brain areas comprising DMN and salience network/ paralimbic regions including anterior temporal cortex, medial prefrontal cortex, dorsal anterior cingulate, temporoparietal junction, and posterior cingulate cortex. These effects are consistent with prior work implicating circuits involved in emotional processing and salience detection, as well as recent reports that demonstrate abnormalities in dynamic shifts between default mode and task-positive networks among those with psychopathic traits (Freeman et al., 2015). Close examination of these effects with consideration of their contrast elements have potentially revelatory implications for models of attention and basic stimulus processing in psychopathy. These effects may demonstrate a physiological mechanism 

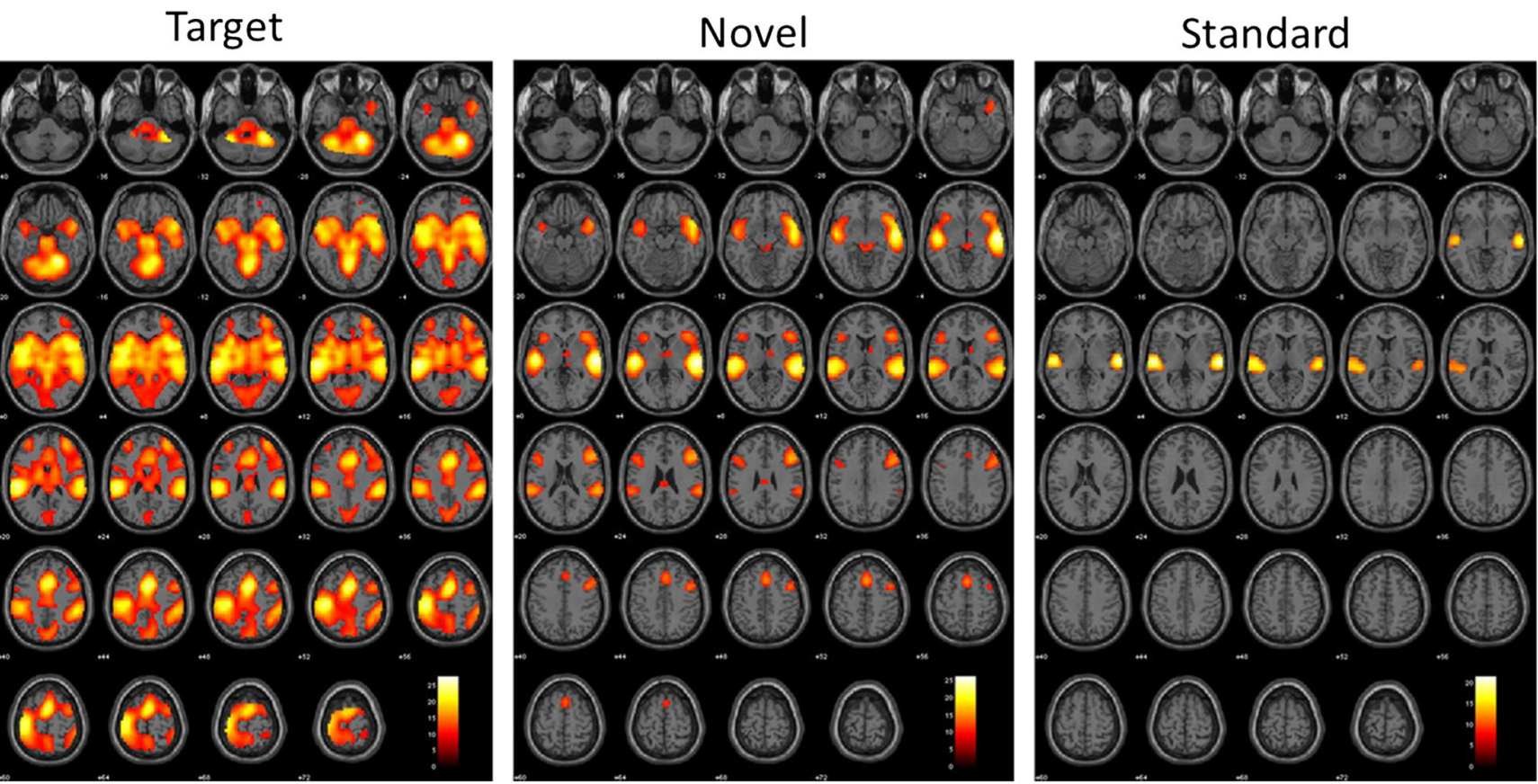

Figure 1. Main effects for target, novel, and standard stimuli. Thresholds are set at $t \geq 10$ to achieve adequate separation. BOLD effects for target and novel stimuli use the standard stimulus as a baseline. The standard stimulus is compared against an implicit (mean) baseline of unmodeled activity

to explain psychopaths' fundamental abnormalities in attention allocation and salience detection, which include diminished processing of stimuli with little motivational significance.

\section{Target and novel effects}

Elevated Factor 1 scores were modestly associated with faster response times for targets. This could reasonably be explained by theoretical perspectives of enhanced approach motivation and reduced inhibitory modulation (Arnett, Smith, \& Newman, 1997). This observation was not accompanied by neural effects systematically related to psychopathy, however. Furthermore, this task was not designed to investigate these effects, so we will defer any speculation or interpretation. However, see our prior work examining neural effects of performance monitoring in a go/no-go task designed to elicit meaningful differences in behavior (Maurer et al., 2015, 2016; Steele et al., 2016).

Table 4. Standard stimuli main effects in ROIs

\begin{tabular}{llllll}
\hline & & \multicolumn{2}{l}{ MNI coordinates } & $t$-value (peak) \\
\hline & & $\mathrm{x}$ & $\mathrm{y}$ & $\mathrm{z}$ & \\
1. & L. Superior Temporal Gyrus & -44 & -36 & 8 & 15.03 \\
2. & R. Superior Temporal Gyrus & 56 & -28 & 4 & 18.55 \\
3. & L. Anterior Cingulate & -24 & 36 & 0 & na \\
\hline
\end{tabular}

Threshold for $p<0.05$ FDR corrected $t=2.91 ; p<0.01 t=3.42$; $p<$ $0.001 t=4.08$
Examining neural activity associated with Target vs. Standard and Novel vs. Standard contrasts, main effects across all subjects demonstrated significant BOLD response in all regions of interest identified previously from $n=100$ healthy controls (Kiehl et al., 2005). Contrary to our expectations, BOLD response for these contrasts revealed relatively sparse correlations with psychopathic traits, and these did not survive strict corrections for multiple comparisons across ROIs. Correlations with individual factors and facets of the PCL-R were primarily positive, especially those relating to PCL-R Factor 1 representing the emotional/interpersonal dimensions of psychopathy.

These findings should not be interpreted as directly contradicting well-replicated ERP findings reporting psychopathyrelated electrocortical effects (see also Anderson et al., 2015). ERP signals result from rapid fluctuations of the brain's electrical activity, whereas BOLD signal measured in fMRI depends on relatively slower hemodynamic activity. As such, it is oversimplistic to conflate BOLD signal with any individual ERP component, particularly ERPs of higher frequency and lower amplitude. These effects are dependent on modelingtask contrasts as discussed in Methods.

Close examination of task conditions reveal that these positive associations are influenced by effects evident in the frequent standard condition, used as a baseline for this contrast. The increased net difference driven by a relatively diminished baseline (also systematically related to Factor 1) and the target activation will present as a positive correlation in this analysis. The effects presented using the standard-stimulus baseline in the target and novel conditions are small, however, and do not 
Table 5. Target stimuli correlations with PCL-R scores with and without covariates in ROIs

\begin{tabular}{|c|c|c|c|c|c|c|c|c|c|c|}
\hline & \multicolumn{3}{|c|}{ MNI coordinates } & \multirow[t]{2}{*}{ PCL-R total } & \multirow[t]{2}{*}{ Factor 1} & \multirow[t]{2}{*}{ Factor 2} & \multirow[t]{2}{*}{ Facet 1} & \multirow[t]{2}{*}{ Facet 2} & \multirow[t]{2}{*}{ Facet 3} & \multirow[t]{2}{*}{ Facet 4} \\
\hline PCL-R scores entered alone in model & $\mathrm{x}$ & $\mathrm{y}$ & $\mathrm{z}$ & & & & & & & \\
\hline R. inferior frontal gyrus & 52 & 8 & 16 & & & & & & & $* 2.96(-)$ \\
\hline L. thalamus/caudate & -16 & -20 & 8 & & $* 2.69(+)$ & & $* 3.03(+)$ & & & \\
\hline L. putamen/globus pallidus & -32 & -4 & -12 & & $* 3.40(+)$ & & $* 3.16(+)$ & $* 3.04(+)$ & & \\
\hline Brainstem & 0 & -24 & -24 & & $* 3.02(+)$ & & $* 2.76(+)$ & & & \\
\hline \multicolumn{11}{|c|}{ PCL-R scores controlling for SD, age, and other factors/facets } \\
\hline R. insula & 44 & 16 & -16 & & & $* 2.44(-)$ & & & & $* * 3.17(-)$ \\
\hline R. precentral gyrus & 28 & -4 & 60 & & & $* 2.81(+)$ & & & $* 3.16(+)$ & \\
\hline L. putamen/globus pallidus & -32 & -4 & -12 & & $* * 3.49(+)$ & & & & & \\
\hline Brainstem & 0 & -24 & -24 & & $* 2.69(+)$ & & & & & \\
\hline
\end{tabular}

$t$-values provided for significant ROIs; $* p<0.05, * * p<0.01$; FDR corrected for ROI volume; $(+)$ indicates positive correlation; $(-)$ indicates negative correlation

survive statistical corrections for multiple tests at ROIs. Furthermore, exploratory analyses utilizing an implicit baseline (in place of the frequent standard condition) did not reveal any new significant effects related to PCL-R scores and largely diminished effects noted. These findings merit close consideration of dynamics influencing the frequent standard condition.

\section{Standard vs. Baseline}

As expected, main effects for the Standard vs. Baseline contrast largely reflect activity in auditory processing areas around the posterior superior temporal gyrus. Kiehl et al. (2005) also reported small activations in frontal/cingulate cortex, which were not replicated here. This may be due to the

Table 6. Novel stimuli correlations with PCL-R scores (with and without other covariates) in ROIs

\begin{tabular}{|c|c|c|c|c|c|c|c|c|c|c|}
\hline \multirow[b]{2}{*}{ PCL-R scores entered alone in model } & \multicolumn{3}{|c|}{ MNI Coordinates } & \multirow[t]{2}{*}{ PCL-R total } & \multirow[t]{2}{*}{ Factor 1} & \multirow[t]{2}{*}{ Factor 2} & \multirow[t]{2}{*}{ Facet 1} & \multirow[t]{2}{*}{ Facet 2} & \multirow[t]{2}{*}{ Facet 3} & \multirow[t]{2}{*}{ Facet 4} \\
\hline & $\mathrm{x}$ & $\mathrm{y}$ & $\mathrm{z}$ & & & & & & & \\
\hline L. inferior frontal gyrus/insula & -44 & 20 & -12 & & & & $* 3.67(+)$ & & & \\
\hline Medial frontal gyrus & 0 & 24 & 36 & & & & & & & $* * 3.79(+)$ \\
\hline R. anterior cingulate gyrus & 4 & 28 & 32 & & & & & & & $* * 4.01(+)$ \\
\hline L. insula & -48 & -8 & -4 & $* 3.22(+)$ & $+2.71(+)$ & & & $* 2.89(+)$ & & \\
\hline R. middle temporal gyrus & -56 & 0 & -16 & $* 3.07(+)$ & & & & & $+3.10(+)$ & \\
\hline L. lingual gyrus/cuneus & -4 & -76 & 12 & & & & & $* 2.59(-)$ & & \\
\hline R. lingual gyrus/cuneus & 4 & -76 & 24 & & $* 2.69(-)$ & & & $* * 3.38(-)$ & & \\
\hline R. thalamus/caudate & 12 & -8 & 8 & $* 2.90(+)$ & & $* 2.83(+)$ & & & & $* 2.95(+)$ \\
\hline R. cerebellum & 16 & -72 & -32 & & & $* 2.56(+)$ & & & & \\
\hline R. cerebellum & 20 & -72 & -28 & & & $* 2.77(+)$ & & & $* 2.66(+)$ & \\
\hline \multicolumn{11}{|c|}{ PCL-R scores controlling for SD, age, and other factors/facets } \\
\hline L. inferior frontal gyrus/insula & -44 & 20 & -12 & & & & $* 3.42(+)$ & & & \\
\hline Medial frontal gyrus & 0 & 24 & 36 & & & & & & & $* * 4.43(+)$ \\
\hline L. anterior cingulate gyrus & 0 & 16 & 32 & & & & & & & *2.91 (+) \\
\hline R. anterior cingulate gyrus & 4 & 28 & 32 & & & & & & & $* * 4.46(+)$ \\
\hline R. middle temporal gyrus & -56 & 0 & -16 & $* 3.05(+)$ & & & & & & \\
\hline L. lingual gyrus/cuneus & -4 & -76 & 12 & & & & & $* 2.64(-)$ & & \\
\hline R. lingual gyrus/cuneus & 4 & -76 & 24 & & & & & $* * 3.24(-)$ & & \\
\hline R. thalamus/caudate & 12 & -8 & 8 & & & & & & & $* 2.52(+)$ \\
\hline
\end{tabular}

T-values provided for significant ROIs; $* p<0.05, * * p<0.01$; FDR corrected for ROI volume; $(+)$ indicates positive correlation; (-) indicates negative correlation 
Table 7. Standard stimuli correlations with PCL-R scores (with and without other covariates) in ROIs

\begin{tabular}{|c|c|c|c|c|c|c|c|c|c|c|}
\hline \multirow[b]{2}{*}{ PCL-R scores entered alone in model } & \multicolumn{3}{|c|}{ MNI Coordinates } & \multirow[t]{2}{*}{ PCL-R total } & \multirow[t]{2}{*}{ Factor 1} & \multirow[t]{2}{*}{ Factor 2} & \multirow[t]{2}{*}{ Facet 1} & \multirow[t]{2}{*}{ Facet 2} & \multirow[t]{2}{*}{ Facet 3} & \multirow[t]{2}{*}{ Facet 4} \\
\hline & $\mathrm{x}$ & $\mathrm{y}$ & $\mathrm{z}$ & & & & & & & \\
\hline L. superior temporal gyrus & -44 & -36 & 8 & $* 2.90(-)$ & $* 2.88(-)$ & & & $* 2.59(-)$ & *2.99 (-) & \\
\hline R. superior temporal gyrus & 56 & -28 & 4 & & $* * 3.67(-)$ & & $* 3.01(-)$ & $* 3.51(-)$ & & $* 2.92(+)$ \\
\hline L. anterior cingulate & -24 & 36 & 0 & & & & & & $* * 3.94(-)$ & \\
\hline \multicolumn{11}{|c|}{ PCL-R scores controlling for SD, age, and other factors/facets } \\
\hline L. superior temporal gyrus & -44 & -36 & 8 & & & & & & & \\
\hline R. superior temporal gyrus & 56 & -28 & 4 & & $* * 4.59(-)$ & $* 2.76(+)$ & & $* 3.15(-)$ & & $* 3.34(+)$ \\
\hline L. anterior cingulate & -24 & 36 & 0 & & $* 2.71(-)$ & & & & & $* 3.22(+)$ \\
\hline
\end{tabular}

$t$-values provided for significant ROIs; ${ }^{*} p<0.05,{ }^{* *} p<0.01$; FDR corrected for ROI volume; (+) indicates positive correlation; (-) indicates negative correlation

significant interactions with PCL-R scores in frontal/cingulate cortex observed.

The Standard vs. Baseline contrast revealed the largest and most stable effects for BOLD activity systematically related to PCL-R scores. This aligns with the size and stability of psychopathy-related effects we recently reported in the large-amplitude slow-wave component elicited by frequent standard stimuli when measured with ERP analyses (Anderson et al., 2015). In the current study, modest associations were present in all ROIs for this contrast, demonstrating significant interactions with PCL-R scores in these regions. Notably, Factor 1 (Facets $1 \&$ 2) and Factor 2 (Facets $3 \& 4$ ) exhibit effects in opposite directions, when accounted for individually. Divergent or unique effects specific to traits corresponding to individual factors and facets of psychopathy have been an increasingly common observation in reports that examine these traits separately (Anderson et al., 2015; Anderson \& Stanford, 2012; Carlson \& Thái, 2010; Carlson, Thái, \& McLarnon, 2009; Juárez et al., 2013; Maurer et al., 2015; Philippi et al., 2015; Pasion, Fernandes, Pereira, \& Barbosa, 2017; Steele et al., 2016; Venables \& Patrick, 2014; Wolf et al., 2015).

Beyond ROIs established for main effects, widespread negative associations that survive whole-brain statistical corrections were observed for PCL-R Factor 1 in areas that include bilateral anterior temporal cortex, medial prefrontal, amygdala, hippocampal, insula, dorsal anterior cingulate, temporoparietal junction, and posterior cingulate regions. All these regions have been previously implicated in the pathophysiology underlying psychopathy. These areas correspond, in part, to paralimbic regions, long implicated in psychopathy (Kiehl, 2006) - or in terms of network models of attention, they include the salience network, as well as regions comprising the DMN.

A close consideration of the task contrasts is important for understanding these effects. Negative correlations in the Standard vs. Baseline contrast demonstrates a net reduction in difference between the standard stimulus and the implicit baseline, systematically related to PCL-R Factor 1. Main effects for the standard condition are primarily dominated by auditory activation (Figure 1) and the implicit baseline condition is comprised of activity in DMN. A relatively persistent default mode and relatively reduced activity in the salience network, which serves to divert processing resources from DMN (Goulden et al., 2014), would combine to produce the smaller net differences, systematically related to Factor 1 (Figure 2). These effects further support network-based analyses that have demonstrated higher power of DMN among psychopaths (Juárez et al., 2013; Freeman et al., 2015) and altered connectivity involving parts of DMN and salience network (Philippi et al., 2015).

\section{Consequences of impaired default mode, salience network function}

Dysfunction in limbic and paralimbic brain areas is commonly associated with psychopathy (Kiehl, 2006; Anderson \& Kiehl, 2012). These regions are usually studied in the context of affect-laden paradigms (Kiehl et al., 2001), and they play an important role in the integration of emotionally salient information into behavioral adaptations (Birbaumer et al., 2005) and higher-order cognitive processes (Blair, 2007). Dysfunction in this context is mostly straightforward; certain affective deficits are paramount among those that characterize psychopathy. The fact that these regions are also implicated here in an elementary component of a basic attention-task, devoid of strong affective content is quite compelling in the context of information-processing models of psychopathy. Reduced amplitude of slow-wave ERPs elicited by frequent standard stimuli have previously been reported in patients with temporoparietal damage (Verleger, Heide, Butt, \& Kompf, 1994). The relevance of temporal lobe dysfunction to psychopathy has a prominent standing in extant literature, including the paralimbic dysfunction model (Kiehl, 2006). In terms of network models serving domains of attention and cognitive function, paralimbic areas overlap significantly 


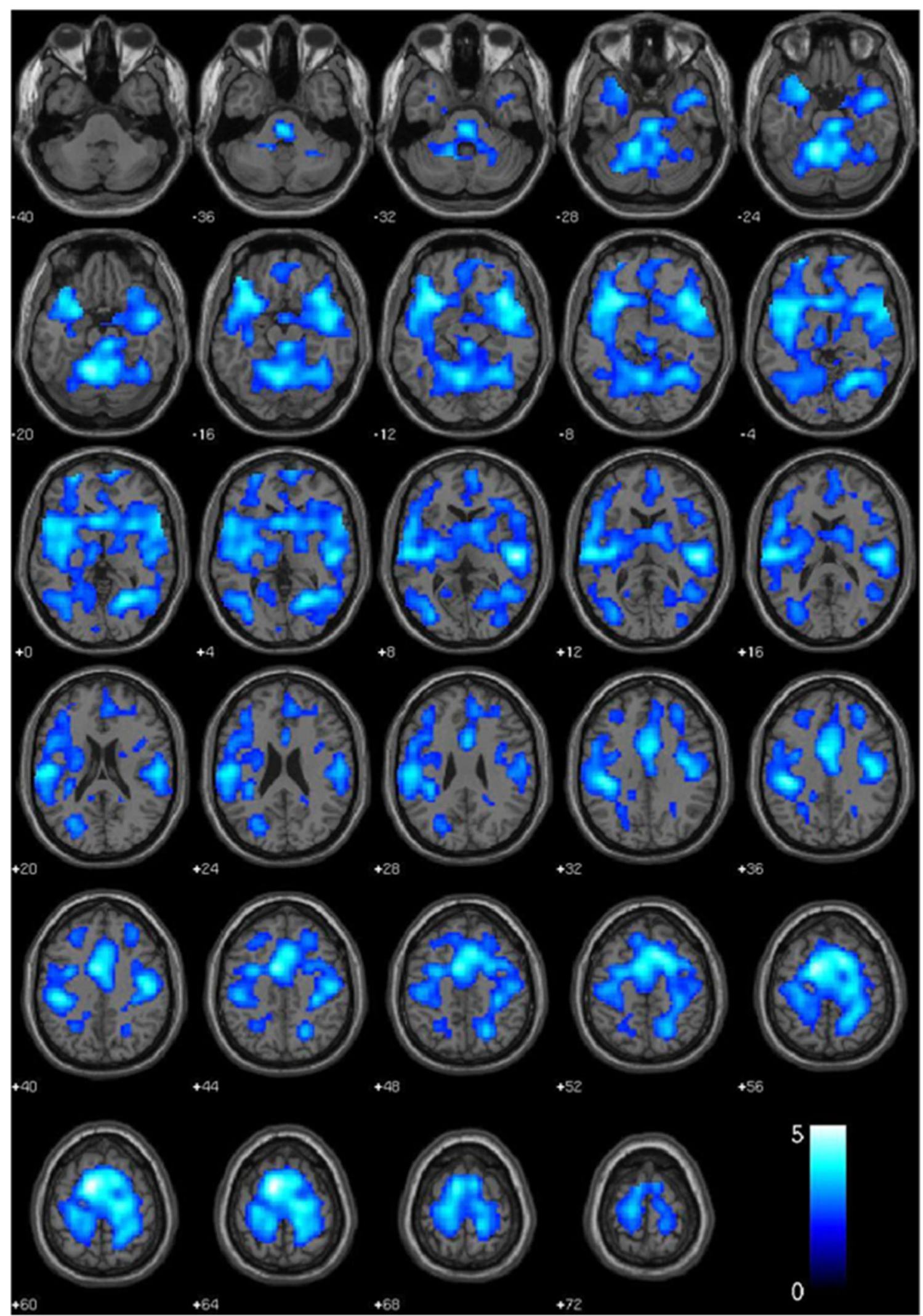

Figure 2. PCL-R Factor 1 negative correlation with standard (vs. baseline) activation. Model controls for the effects of age, substance dependence, and PCL-R Factor 2. Effects are significant at threshold of $p<.05$, corrected for False Discovery Rate (FDR) across the whole brain 
Table 8. Standard stimuli correlations with PCL-R Factor 1 scores across the entire brain

\begin{tabular}{|c|c|c|c|c|c|}
\hline & $\mathrm{BA}$ & $\mathrm{X}$ & $\mathrm{Y}$ & Z & $t$-value \\
\hline \multicolumn{6}{|l|}{ Frontal Lobes } \\
\hline 1. L. Superior frontal gyrus $* * *$ & 6 & -9 & 3 & 63 & 5.17 \\
\hline 2. R. Superior frontal gyrus $* * *$ & 6 & 15 & 9 & 54 & 4.65 \\
\hline 3. L. Superior frontal gyrus** & 10 & -27 & 57 & -3 & 3.37 \\
\hline 4. R. Inferior frontal gyrus $* * *$ & 47 & 39 & 18 & -12 & 4.54 \\
\hline 5. L. Inferior frontal gyrus $* * *$ & 47 & -30 & 18 & -12 & 4.50 \\
\hline 6. R. Precentral gyrus $* * *$ & 6 & 48 & -18 & 39 & 4.16 \\
\hline 7. L. Precentral gyrus*** & 4 & -24 & -24 & 66 & 3.78 \\
\hline 8. L. Precentral gyrus*** & 4 & -15 & -21 & 69 & 3.78 \\
\hline \multicolumn{6}{|l|}{ Parietal lobes } \\
\hline 9. L. Postcentral gyrus $* * *$ & 2 & -42 & -27 & 36 & 4.48 \\
\hline 10. L. Postcentral gyrus $* * *$ & 32 & -54 & -18 & 39 & 4.37 \\
\hline \multicolumn{6}{|l|}{ Temporal lobes } \\
\hline 11. R. Transverse temporal gyrus*** & 41 & 51 & -21 & 9 & 4.84 \\
\hline 12. R. Superior temporal gyrus*** & 38 & 45 & 0 & -18 & 4.20 \\
\hline 13. R. Superior temporal gyrus $* * *$ & 22 & 51 & 6 & -9 & 4.07 \\
\hline 14. R. Middle temporal gyrus** & 37 & 42 & -57 & 0 & 3.69 \\
\hline \multicolumn{6}{|l|}{ Limbic } \\
\hline 15. L. Anterior cingulate*** & 24 & -6 & 3 & 42 & 4.71 \\
\hline 16. L. Insula*** & 13 & -36 & -9 & -3 & 4.17 \\
\hline 17. L. Parahippocampal gyrus $* * *$ & 34 & -27 & 6 & -21 & 4.07 \\
\hline 18. R. Parahippocampal gyrus*** & 19 & 33 & -54 & -9 & 3.80 \\
\hline \multicolumn{6}{|l|}{ Occipital } \\
\hline 19. R. Lingual gyrus*** & 19 & 24 & -63 & 0 & 4.38 \\
\hline 20. L. Lingual gyrus* & 17 & -6 & -93 & -3 & 2.30 \\
\hline 21. L. Middle occipital** & 19 & -33 & -78 & 6 & 3.45 \\
\hline 22. L. Middle occipital** & 19 & -39 & -66 & 3 & 3.42 \\
\hline \multicolumn{6}{|l|}{ Subcortical } \\
\hline 23. L. Claustrum*** & NA & -36 & -18 & 9 & 3.99 \\
\hline 24. L. Caudate head $* * *$ & NA & -6 & 15 & 0 & 3.92 \\
\hline 25. R. Brainstem*** & NA & 3 & -30 & -15 & 3.84 \\
\hline \multicolumn{6}{|l|}{ Cerebellum } \\
\hline 26. L. Culmen*** & NA & -12 & -48 & -24 & 4.63 \\
\hline 27. R. Culmen*** & NA & 6 & -27 & -24 & 4.01 \\
\hline 28. L. Culmen**** & NA & -3 & -60 & -12 & 4.23 \\
\hline 29. R. Lingual*** & NA & 9 & -48 & -21 & 3.93 \\
\hline 30. R. Culmen*** & NA & 3 & -30 & -33 & 3.90 \\
\hline
\end{tabular}

$* * *$ FDR corrected $p<0.005 ; * *$ FDR corrected $p<0.01$; *FDR corrected $p<0.05$

Peak coordinates for clusters are in MNI space, labels from Talairach Daemon nearest gray matter

Covariates of age, substance dependence, and Factor 2 are included in the model

with the salience network and DMN. The dynamic interplay between these two networks has been the subject of many recent investigations of normal and disordered cognitive functions.
The salience network comprises dorsal anterior cingulate and fronto-anterior insular cortex, supported by dense connectivity to primary limbic structures (Seeley et al., 2007). It has been demonstrated to regulate dynamic shifts between the default mode and externally focused attention networks (Goulden et al., 2014; Menon \& Uddin, 2010; Sridharan, Levitin, \& Menon, 2008). In fact, damage to these circuits reliably predicts deficiencies in appropriate deactivation DMN and is associated with behavioral consequences such as failures of inhibition (Bonnelle et al., 2012; Jilka et al., 2014). The dynamic interplay between default mode and salience network activation is thus a reasonable platform from which to examine fundamental attention-based deficits in psychopath that demonstrably scale up to classic behavioral deficits in psychopathy including impaired inhibition (Bonnelle et al., 2012), diminished error processing (Ham, Leff, de Boissezon, Joffe, \& Sharp, 2013), weak affective processing (Anderson et al., 2017; Fichtenholtz et al., 2004; Lane et al., 1998), and abnormal patterns of moral judgement (Chiong et al., 2013).

Insofar as these networks work together in harnessing attentional resources for the basic encoding of motivationally relevant stimuli, the present findings support accumulating research demonstrating fundamental deficits in basic cognitive systems in psychopathy. A recent extension of a prominent attention-based model of psychopathy, the impaired integration model, has highlighted deficits in the DMN and salience network in psychopathy (Hamilton et al., 2015). It is suggested that disturbances between networks impair rapid integration and adaptation to fluctuating priorities in the environment. Scaling this up to higher-order cognition, these failures would hypothetically degrade the facile prioritization of affective information in complex environments with many competing domains of information. Further, in a long-range developmental context, these failures could ultimately undermine the natural salience of emotionally relevant information. The present findings add to this literature by demonstrating hemodynamic patterns consistent with impaired shifts between networks in an event-related, nonaffective fMRI paradigm.

\section{Limitations and future directions}

This study examines the neuroanatomical correlates of certain fundamental abnormalities in information processing that have long been recognized in psychopathy. While a rich literature precedes this report examining oddball target detection in psychopathy using ERPs (Gao \& Raine, 2009), this is the first report examining these effects using traditional contrastdriven event-related fMRI. ERPs and fMRI are each endowed with inherent strengths and limitations. While fMRI promotes more specific neuroanatomical precision than ERPs it does not approach the same temporal specificity. The present study 
importantly implicates brain regions supporting default mode and salience network function, and the event-related contrasts provide valuable task-related specificity for abnormal hemodynamic response. Additional specificity may be gained if future studies were to implement multi-modal fusion, relating ERP data directly to fMRI measures (Calhoun et al., 2006).

This study also examined a rather limited segment of the population, adult male inmates. Inmates have traditionally been used to study the pathological extremes on dimensions of psychopathic traits, because the incidence of clinically relevant psychopathic traits among incarcerated males is approximately 20 times higher than among nonincarcerated samples (Hare, 2003). Still, psychopathic traits are increasingly studied on a continuum (Hare \& Neumann, 2005; Walters, Duncan, \& Mitchell-Perez, 2007), and nonincarcerated samples also have revealed valuable insights into the etiology and pathophysiology of psychopathy (DeMatteo, Helibrun, \& Marczyk, 2006; Gao \& Raine, 2010). Furthermore, the incidence and presentation of psychopathy among women is still being clarified, and the examination of psychopathic traits among juveniles is rapidly expanding. The extension of the present work to more diverse samples that include females and juveniles, as well as nonincarcerated community samples is an important next step.

Finally, these findings speak to rather specific, fundamental elements of cognitive processing in psychopathy, i.e., networks that support the mobilization and flexibility of attention. The simplicity of this auditory oddball task serves to demonstrate functional abnormalities in nonaffective contexts, and the results reveal important guides for future research to build on. However, the methods that we used to exploit certain functional properties of the brain inherently limit the generalization of these findings without further replication and extension. It will be important that extensions of this work, examining more complex cognitive processes serving emotion, inhibition, moral processing, etc. consider these effects along with prevailing differences in the mobilization of attention that likely cut across these tasks and importantly influence contrast-driven, even-related $\mathrm{fMRI}$ results.

\section{Summary and conclusions}

Certain affective deficits have been recognized as definitive features of psychopathy since the honing of its modern clinical description (Cleckley, 1941). Progress in cognitive neuroscience has added a more thorough understanding of these features, especially the physiological correlates, cognitive contexts, and boundaries that limit affective processing among psychopaths (Anderson \& Kiehl, 2012; Kiehl, 2006). Emerging evidence suggests that some of these limitations are precipitated by fundamentally impaired mechanisms of attention that hinder flexible distribution of cognitive resources (Hamilton et al., 2015). The data presented demonstrate that these abnormalities are apparent in hemodynamic neural responses during a simple auditory oddball task. Psychopathy-related differences are most prominent when comparing baseline neural activity to the most elementary task-related events (i.e., the frequent standard stimulus). In the context of network models of attention, these findings suggest relatively reduced differences in activation between DMN and salience network while shifts of attention to taskrelevant, external stimuli should be engaged more readily. This is hypothetically detrimental to several higher-order processes deficient in psychopathy including inhibition (Bonnelle et al., 2012), error processing (Ham et al., 2013), affective processing (Fichtenholtz et al., 2004), and moral judgement (Chiong et al., 2013). As such, this model remains a compelling platform from which to examine the pathophysiology underlying psychopathy.

Acknowledgements The authors thank Keith Harenski and Prashanth Nyalakanti for their assistance in data analysis. They also thank the Mind Research Network and the New Mexico Department of Corrections for their cooperative efforts. This research was supported in part by the National Institute of Mental Health R01 MH070539 (PI: Kiehl) and F32MH098532 (PI: Anderson).

\section{References}

Anderson, N. E., \& Kiehl, K. A. (2012). The psychopath magnetized: insights from brain imaging. Trends in cognitive sciences, 16(1), 5260. https://doi.org/10.1016/j.tics.2011.11.008

Anderson, N. E., \& Stanford, M. S. (2012). Demonstrating emotional processing differences in psychopathy using affective ERP modulation. Psychophysiology, 49(6), 792-806. https://doi.org/10.1111/j. 1469-8986.2012.01369.x

Anderson, N. E., Steele, V. R., Maurer, J. M., Bernat, E. M., \& Kiehl, K. A. (2015). Psychopathy, attention, and oddball target detection: New insights from PCL-R facet scores. Psychophysiology, 52(9), 11941204. https://doi.org/10.1111/psyp.12441

Anderson, N. E., Steele, V. R., Maurer, J. M., Rao, V., Koenigs, M. R., Decety, J., ... Kiehl, K. A. (2017). Differentiating emotional processing and attention in psychopathy with functional neuroimaging. Cognitive, Affective, \& Behavioral Neuroscience, 17(3), 491-515. https://doi.org/10.3758/s13415-016-0493-5

Arnett, P. A., Smith, S. S., \& Newman, J. P. (1997). Approach and avoidance motivation in psychopathic criminal offenders during passive avoidance. Journal of personality and social psychology, 72(6), 1413. https://doi.org/10.1037/0022-3514.72.6.1413

Baskin-Sommers, A., Curtin, J. J., Li, W., \& Newman, J. P. (2012). Psychopathy-related differences in selective attention are captured by an early event-related potential. Personality Disorders: Theory, Research, and Treatment, 3(4), 370-378. https://doi.org/10.1037/ a0025593

Baskin-Sommers, A. R., Curtin, J. J., \& Newman, J. P. (2011). Specifying the attentional selection that moderates the fearlessness of psychopathic offenders. Psychological Science, 22(2), 226-234. https://doi. org/10.1177/0956797610396227

Baskin-Sommers, A. R., Curtin, J. J., \& Newman, J. P. (2013). Emotionmodulated startle in psychopathy: Clarifying familiar effects. Journal of Abnormal Psychology, 122(2), 458-468. https://doi.org/ 10.1037/a0030958 
Birbaumer, N., Veit, R., Lotze, M., Erb, M., Hermann, C., Grodd, W., \& Flor, H. (2005). Deficient fear conditioning in psychopathy: a functional magnetic resonance imaging study. Archives of General Psychiatry, 62(7), 799-805. https://doi.org/10.1001/archpsyc.62.7. 799

Blair, R. J. R. (2005). Applying a cognitive neuroscience perspective to the disorder of psychopathy. Development and psychopathology, 17(03), 865-891. https://doi.org/10.1017/S0954579405050418

Blair, R. J. R. (2007). The amygdala and ventromedial prefrontal cortex in morality and psychopathy. Trends in cognitive sciences, 11(9), 387392. https://doi.org/10.1016/j.tics.2007.07.003

Blair, R. J. R., Peschardt, K. S., Budhani, S., Mitchell, D. G. V., \& Pine, D. S. (2006). The development of psychopathy. Journal of Child Psychology and Psychiatry, 47(3-4), 262-276. https://doi.org/10. $1111 / j .1469-7610.2006 .01596 . x$

Bonnelle, V., Ham, T. E., Leech, R., Kinnunen, K. M., Mehta, M. A., Greenwood, R. J., \& Sharp, D. J. (2012). Salience network integrity predicts default mode network function after traumatic brain injury. Proceedings of the National Academy of Sciences, 109(12), 46904695. https://doi.org/10.1073/pnas.1113455109

Calhoun, V. D., Adali, T., Pearlson, G. D., \& Kiehl, K. A. (2006). Neuronal chronometry of target detection: fusion of hemodynamic and event-related potential data. Neuroimage, 30(2), 544-553. https://doi.org/10.1016/j.neuroimage.2005.08.060

Calhoun, V. D., Kiehl, K. A., \& Pearlson, G. D. (2008). Modulation of temporally coherent brain networks estimated using ICA at rest and during cognitive tasks. Human Brain Mapping, 29(7), 828-838. https://doi.org/10.1002/hbm.20581

Calhoun, V. D., Stevens, M. C., Pearlson, G. D., \& Kiehl, K. A. (2004). fMRI analysis with the general linear model: removal of latencyinduced amplitude bias by incorporation of hemodynamic derivative terms. Neuroimage, 22(1), 252-257. https://doi.org/10.1016/j. neuroimage.2003.12.029

Carlson, S. R. \& Thái, S. (2010). ERPs on a continuous performance task and self-reported psychopathic traits: $\mathrm{P} 3$ and $\mathrm{CNV}$ augmentation are associated with Fearless Dominance. Biological Psychology, 85(2), 318-330. https://doi.org/10.1016/j.biopsycho.2010.08.002

Carlson, S. R., Thái, S., \& McLarnon, M. E. (2009). Visual P3 amplitude and self-reported psychopathic personality traits: Frontal reduction is associated with self-centered impulsivity. Psychophysiology, 46(1), 100-113. https://doi.org/10.1111/j.1469-8986.2008.00756.x

Chiong, W., Wilson, S. M., D'Esposito, M., Kayser, A. S., Grossman, S. N., Poorzand, P., ... Rankin, K. P. (2013). The salience network causally influences default mode network activity during moral reasoning. Brain, 136(6), 1929-1941. https://doi.org/10.1093/brain/ awt066

Cleckley, H. (1941). The Mask of Sanity. St. Louis, MO: Mosby.

Cocchi, L., Zalesky, A., Fornito, A., \& Mattingley, J. B. (2013). Dynamic cooperation and competition between brain systems during cognitive control. Trends in Cognitive Sciences, 17, 493-501. https://doi. org/10.1016/j.tics.2013.08.006

Cope, L. M., Vincent, G. M., Jobelius, J. L., Nyalakanti, P. K., Calhoun, V. D., \& Kiehl, K. A. (2014). Psychopathic traits modulate brain responses to drug cues in incarcerated offenders. Frontiers in Human Neuroscience, 8, 1-16 https://doi.org/10.3389/fnhum.2014. 00087

DeMatteo, D., Heilbrun, K., \& Marczyk, G. (2006). An empirical investigation of psychopathy in a noninstitutionalized and noncriminal sample. Behavioral Sciences \& the Law, 24(2), 133-146. https:// doi.org/10.1002/bsl.667

Ermer, E., Cope, L. M., Nyalakanti, P. K., Calhoun, V. D., \& Kiehl, K. A. (2012). Aberrant paralimbic graymatter in criminal psychopathy. Journal of Abnormal Psychology, 121(3), 649-658. https://doi.org/ 10.1037/a0026371

Fichtenholtz, H. M., Dean, H. L., Dillon, D. G., Yamasaki, H., McCarthy, G., \& LaBar, K. S. (2004). Emotion-attention network interactions during a visual oddball task. Cognitive Brain Research, 20(1), 6780. https://doi.org/10.1016/j.cogbrainres.2004.01.006

First, M.B., Spitzer, R.L., Gibbon, M., Williams, J.B.W. (2002). Structured Clinical Interview for DSM-IV-TR Axis I Disorders, Research Version, Patient Edition (SCID-I/P). New York, NY: Biometrics Research, New York State Psychiatric Institute.

Fox, M. D., Snyder, A. Z., Vincent, J. L., Corbetta, M., Van Essen, D. C., \& Raichle, M. E. (2005). The human brain is intrinsically organized into dynamic, anticorrelated functional networks. Proceedings of the National Academy of Sciences of the United States of America, 102(27), 9673-9678. https://doi.org/10.1073/pnas.0504136102

Freeman, S. M., Clewett, D. V., Bennett, C. M., Kiehl, K. A., Gazzaniga, M. S., \& Miller, M. B. (2015). The posteromedial region of the default mode network shows attenuated task-induced deactivation in psychopathic prisoners. Neuropsychology, 29(3), 493. https://doi. org/10.1037/neu0000118

Freire, L., Mangin, J.F., 2001. Motion correction algorithms may create spurious brain activations in the absence of subject motion. NeuroImage 14 (3), 709 - 722. https://doi.org/10.1006/nimg.2001. 0869

Freire, L., Roche, A., \& Mangin, J. F. (2002). What is the best similarity measure for motion correction in fMRI time series?. IEEE transactions on medical imaging, 21(5), 470-484. https://doi.org/10.1109/ TMI.2002.1009383

Frick, P. J., \& White, S. F. (2008). Research review: The importance of callous-unemotional traits for developmental models of aggressive and antisocial behavior. Journal of Child Psychology and Psychiatry, 49(4), 359-375. https://doi.org/10.1111/j.1469-7610. 2007.01862.x

Friston, K., Ashburner, J., Frith, C. D., Poline, J. B., Heather, J. D., \& Frackowiak, R. S. (1995). Spatial registration and normalization of images. Human brain mapping, 3(3), 165-189. https://doi.org/10. 1002/hbm.460030303

Gao, Y., \& Raine, A. (2009). P3 event-related potential impairments in antisocial and psychopathic individuals: A meta-analysis. Biological Psychology, 82(3), 199-210.

Gao, Y., \& Raine, A. (2010). Successful and unsuccessful psychopaths: A neurobiological model. Behavioral sciences \& the law, 28(2), 194210. https://doi.org/10.1016/j.biopsycho.2009.06.006

García-Larrea, L., \& Cézanne-Bert, G. (1998). P3, positive slow wave and working memory load: a study on the functional correlates of slow wave activity. Electroencephalography and Clinical Neurophysiology/Evoked Potentials Section, 108(3), 260-273. https://doi.org/10.1016/S0168-5597(97)00085-3

Genovese, C. R., Lazar, N. A., \& Nichols, T. (2002). Thresholding of statistical maps in functional neuroimaging using the false discovery rate. Neuroimage, 15(4), 870-878. https://doi.org/10.1006/nimg. 2001.1037

Goulden, N., Khusnulina, A., Davis, N. J., Bracewell, R. M., Bokde, A. L., McNulty, J. P., \& Mullins, P. G. (2014). The salience network is responsible for switching between the default mode network and the central executive network: replication from DCM. Neuroimage, 99, 180-190. https://doi.org/10.1016/j.neuroimage.2014.05.052

Grech, R., Cassar, T., Muscat, J., Camilleri, K. P., Fabri, S. G., Zervakis, M., ... Vanrumste, B. (2008). Review on solving the inverse problem in EEG source analysis. Journal of Neuroengineering and Rehabilitation, 5(1), 25. https://doi.org/10.1186/1743-0003-5-25

Greicius, M. D., Krasnow, B., Reiss, A. L., \& Menon, V. (2003). Functional connectivity in the resting brain: a network analysis of the default mode hypothesis. Proceedings of the National Academy of Sciences, 100(1), 253-258. https://doi.org/10.1073/pnas. 0135058100

Halgren, E., Marinkovic, K., \& Chauvel, P. (1998). Generators of the late cognitive potentials in auditory and visual oddball tasks. Electroencephalography and Clinical Neurophysiology, 106 (2), 156-164. https://doi.org/10.1016/S0013-4694(97)00119-3 
Ham, T., Leff, A., de Boissezon, X., Joffe, A., \& Sharp, D. J. (2013). Cognitive control and the salience network: an investigation of error processing and effective connectivity. Journal of Neuroscience, 33(16), 7091-7098. https://doi.org/10.1523/JNEUROSCI.4692-12. 2013

Hamilton, R. K., Hiatt Racer, K., \& Newman, J. P. (2015). Impaired integration in psychopathy: A unified theory of psychopathic dysfunction. Psychological review, 122(4), 770. https://doi.org/10. 1037/a0039703

Hare R. D. (2003). Manual for the Hare Psychopathy Checklist-Revised (Multi-Health Systems, Toronto, Canada), 3rd Ed.

Hare, R. D. \& Neumann, C. S. (2005). Structural models of psychopathy. Current Psychiatry Reports, 7(1), 57-64. https://doi.org/10.1007/ s11920-005-0026-3

Hare, R. D., \& Neumann, C. S. (2006). The PCL-R assessment of psychopathy: Development, structural properties and new directions. In C.J. Patrick (Ed.), Handbook of Psychopathy (pp. 58-88). New York: Guilford.

Herrmann, C. S. \& Knight, R. T. (2001). Mechanisms of human attention: Event-related potentials and oscillations. Neuroscience \& Biobehavioral Reviews, 25, 465-476. https://doi.org/10.1016/ S0149-7634(01)00027-6

Jilka, S. R., Scott, G., Ham, T., Pickering, A., Bonnelle, V., Braga, R. M., ... Sharp, D. J. (2014). Damage to the salience network and interactions with the default mode network. Journal of neuroscience, 34(33), 10798-10807. https://doi.org/10.1523/JNEUROSCI.051814.2014

Juárez, M., Kiehl, K. A., \& Calhoun, V. D. (2013). Intrinsic limbic and paralimbic networks are associated with criminal psychopathy. Human Brain Mapping, 34(8), 1921-1930. https://doi.org/10.1002/ hbm. 22037

Kiehl, K. A. (2006). A cognitive neuroscience perspective on psychopathy: evidence for paralimbic system dysfunction. Psychiatry Research, 142(2), 107-128. https://doi.org/10.1016/j.psychres. 2005.09 .013

Kiehl, K. A., \& Liddle, P. F. (2001). An event-related functional magnetic resonance imaging study of an auditory oddball task in schizophrenia. Schizophrenia Research, 48(2), 159-171. https://doi.org/10. 1016/S0920-9964(00)00117-1

Kiehl, K. A., Smith, A. M., Hare, R. D., Mendrek, A., Forster, B. B., Brink, J., \& Liddle, P. F. (2001). Limbic abnormalities in affective processing by criminal psychopaths as revealed by functional magnetic resonance imaging. Biological psychiatry, 50(9), 677-684. https://doi.org/10.1016/S0006-3223(01)01222-7

Kiehl, K. A., Stevens, M. C., Laurens, K. R., Pearlson, G., Calhoun, V. D., \& Liddle, P. F. (2005). An adaptive reflexive processing model of neurocognitive function: supporting evidence from a large scale $(\mathrm{n}=100)$ fMRI study of an auditory oddball task. Neuroimage, 25(3), 899-915. https://doi.org/10.1016/j.neuroimage.2004.12.035

Kim, H. (2013). Differential neural activity in the recognition of old versus new events: An Activation Likelihood Estimation MetaAnalysis. Human Brain Mapping, 34(4), 814-836. https://doi.org/ 10.1002/hbm.21474

Lane, R. D., Reiman, E. M., Axelrod, B., Yun, L. S., Holmes, A., \& Schwartz, G. E. (1998). Neural correlates of levels of emotional awareness: Evidence of an interaction between emotion and attention in the anterior cingulate cortex. Journal of Cognitive Neuroscience, 10(4), 525-535. https://doi.org/10.1162/ 089892998562924

Maldjian, J. A., Laurienti, P. J., Kraft, R. A., \& Burdette, J. H. (2003). An automated method for neuroanatomic and cytoarchitectonic atlasbased interrogation of fMRI data sets. Neuroimage, 19(3), 12331239. https://doi.org/10.1016/S1053-8119(03)00169-1

Maurer, J. M., Steele, V. R., Cope, L. M., Vincent, G. M., Stephen, J. M., Calhoun, V. D., \& Kiehl, K. A. (2016). Dysfunctional error-related processing in incarcerated youth with elevated psychopathic traits.
Developmental cognitive neuroscience, 19, 70-77. https://doi.org/ 10.1016/j.dcn.2016.02.006

Maurer, J. M., Steele, V. R., Edwards, B. G., Bernat, E. M., Calhoun, V. D., Kiehl, K. A. (2015). Dysfunctional error-related processing in female psychopathy. Social cognitive and affective neuroscience, 11(7), 1059-1068. https://doi.org/10.1093/scan/nsv070

Mazaika, P., Whitfield-Gabrieli, S., Reiss, A., \& Glover, G. (2007). Artifact repair for fMRI data from high motion clinical subjects. Presented at Human Brain Mapping annual meeting.

Menon, V., \& Uddin, L. Q. (2010). Saliency, switching, attention and control: a network model of insula function. Brain Structure and Function, 214(5-6), 655-667. https://doi.org/10.1007/s00429-0100262-0

Newman, J. P. (1998). Psychopathic behavior: An information processing perspective. In Psychopathy: Theory, research and implications for society (pp. 81-104). Springer Netherlands.

Newman, J. P., Curtin, J. J., Bertsch, J. D., \& Baskin-Sommers, A. R. (2010). Attention moderates the fearlessness of psychopathic offenders. Biological Psychiatry, 67(1), 66-70. https://doi.org/10. 1016/j.biopsych.2009.07.035

Nieuwenhuis, S., Aston-Jones, G., \& Cohen, J. D. (2005). Decision making, the P3, and the locus coeruleus-norepinephrine system. Psychological bulletin, 131(4), 510. https://doi.org/10.1037/00332909.131.4.510

Pasion, R., Fernandes, C., Pereira, M. R., \& Barbosa, F. (2017) Antisocial behaviour and psychopathy: uncovering the externalizing link in the P3 modulation. Neuroscience \& Biobehavioral Reviews, online prior to publication. https://doi.org/10.1016/j.neubiorev. 2017.03.012

Pfefferbaum, A., Ford, J. M., \& Kraemer, H. C. (1990). Clinical utility of long latency 'cognitive' event-related potentials (P3): the cons. Electroencephalography and Clinical Neurophysiology, 76, 6-12. https://doi.org/10.1016/0013-4694(90)90052-L

Philippi, C. L., Pujara, M. S., Motzkin, J. C., Newman, J., Kiehl, K. A., \& Koenigs, M. (2015). Altered resting-state functional connectivity in cortical networks in psychopathy. Journal of Neuroscience, 35(15), 6068-6078. https://doi.org/10.1523/JNEUROSCI.5010-14.2015

Polich, J. (1987). Task difficulty, probability, and inter-stimulus interval as determinants of P300 from auditory stimuli. Electroencephalography and Clinical Neurophysiology/Evoked Potentials Section, 68(4), 311-320. https://doi.org/10.1016/01685597(87)90052-9

Polich, J., \& Kok, A. (1995). Cognitive and biological determinants of P300: an integrative review. Biological Psychology, 41(2), 103-146. https://doi.org/10.1016/0301-0511(95)05130-9

Polich, J. \& Squire, L.R. (1993). P300 from amnesic patients with bilateral hippocampal lesions. Electroencephalography and Clinical Neurphysiology, 86 (6), 408-417. https://doi.org/10.1016/00134694(93)90136-J

Pujol, J., Batalla, I., Contreras-Rodríguez, O., Harrison, B. J., Pera, V., Hernández-Ribas, R., ... López-Solà, M. (2011). Breakdown in the brain network subserving moral judgment in criminal psychopathy. Social cognitive and affective neuroscience, 7(8), 917-923. https:// doi.org/10.1093/scan/nsr075

Raichle, M. E., MacLeod, A. M., Snyder, A. Z., Powers, W. J., Gusnard, D. A., \& Shulman, G. L. (2001). A default mode of brain function. Proceedings of the National Academy of Sciences, 98(2), 676-682. https://doi.org/10.1073/pnas.98.2.676

Seeley, W. W., Menon, V., Schatzberg, A. F., Keller, J., Glover, G. H., Kenna, H., ... Greicius, M. D. (2007). Dissociable intrinsic connectivity networks for salience processing and executive control. Journal of Neuroscience, 27(9), 2349-2356. https://doi.org/10. 1523/JNEUROSCI.5587-06.2007

Sheng, T., Gheytanchi, A., \& Aziz-Zadeh, L. (2010). Default network deactivations are correlated with psychopathic personality traits. 
PloS one, 5(9), e12611. https://doi.org/10.1371/journal.pone. 0012611

Sridharan, D., Levitin, D. J., \& Menon, V. (2008). A critical role for the right fronto-insular cortex in switching between central-executive and default-mode networks. Proceedings of the National Academy of Sciences, 105(34), 12569-12574. https://doi.org/10.1073/pnas. 0800005105

Stark, C. E., \& Squire, L. R. (2001). When zero is not zero: the problem of ambiguous baseline conditions in fMRI. Proceedings of the National Academy of Sciences, 98(22), 12760-12766. https://doi. org/10.1073/pnas.221462998

Steele, V. R., Maurer, J. M., Bernat, E. M., Calhoun, V. D., Kiehl, K. A. (2016). Error-related processing in adult males with elevated psychopathic traits. Personality Disorders: Theory, Research, and Treatment 7(1), 80-90. https://doi.org/10.1037/per0000143

Stevens, M. C., Laurens, K. R., Liddle, P. F., \& Kiehl, K. A. (2006). The hemodynamics of oddball processing during single-tone and twotone target detection tasks. International journal of psychophysiology, 60(3), 292-303. https://doi.org/10.1016/j.jpsycho.2005.07.012

Sullivan, E. A., \& Kosson, D. S. (2006). Ethnic and cultural variations in psychopathy. In C.J. Patrick (Ed.), Handbook of Psychopathy (pp. 437-458). New York: Guilford.

Tzourio-Mazoyer, N., Landeau, B., Papathanassiou, D., Crivello, F., Etard, O., Delcroix, N., ... Joliot, M. (2002). Automated anatomical labeling of activations in SPM using a macroscopic anatomical parcellation of the MNI MRI single-subject brain. Neuroimage, 15(1), 273-289. https://doi.org/10.1006/nimg.2001.0978

Venables, N. C., \& Patrick, C. J. (2014). Reconciling discrepant findings for P3 brain response in criminal psychopathy through reference to the concept of externalizing proneness. Psychophysiology, 51(5), 427-436. https://doi.org/10.1111/psyp.12189

Ventura, J., Liberman, R. P., Green, M. F., Shaner, A., \& Mintz, J. (1998). Training and quality assurance with the Structured Clinical Interview for DSM-IV (SCID-I/P). Psychiatry research, 79(2), 163-173. https://doi.org/10.1016/S0165-1781(98)00038-9

Verleger, R., Heide, W., Butt, C., \& Kompf, D. (1994). Reduction of P3b in patients with temporo-parietal lesions. Cognitive Brain Research, 2, 103-116. https://doi.org/10.1016/0926-6410(94)90007-8

Walters, G. D., Duncan, S. A., \& Mitchell-Perez, K. (2007). The latent structure of psychopathy: A taxometric investigation of the Psychopathy Checklist-Revised in a heterogeneous sample of male prison inmates. Assessment, 14(3), 270-278.

Wechsler, D. A. (1997). Wechsler Adult Intelligence Scale III. San Antonio, TX: The Psychological Corporation.

Wolf, R.C., Pujara, M., Motzkin, J.C., Newman, J. P., Kiehl, K. A., Decety, J., Koenigs, M. (2015). Interpersonal traits of psychopathy linked to reduced integrity of the uncinate fasciculus. Human Brain Mapping, 36(10), 4202-4209. https://doi.org/10.1002/hbm.22911 